\title{
Automatic Detection of Abnormalities Associated with Abdomen and Liver Images: A Survey on Segmentation Methods
}

\author{
G.G. Rajput \\ Department of Computer Science \\ Rani Channamma University \\ Belagavi, India
}

\author{
Anand M. Chavan \\ Department of Computer Science \\ Solapur University \\ Solapur, India
}

\begin{abstract}
Image segmentation plays an important role in medical imaging by automating detection of false structures and other regions of interest. An image segmentation method partitions an image into multiple segments, representing an image into more meaningful, simpler and easier to analyze. Several general-purpose algorithm and techniques have been developed for image segmentation. This paper explains different segmentation techniques used in medical image analysis addressing the segmentation of abdominal and liver images as case study. Experiments are performed on abdominal and liver CT scan images and the outcomes of these segmentation techniques are discussed. Performance of the methods is presented on the basis of parameters namely, pixel values, mean and standard deviation.
\end{abstract}

\section{Keywords}

Segmentation, thresholding, clustering, artificial neural network, edge detection, region of interest

\section{INTRODUCTION}

Digital image processing is the use of computer algorithms for processing digital images. One important application of digital image processing is medical imaging. It is an important tool in medicine. Computer Tomography (CT), Magnetic Resonance Imaging (MRI), Ultra Sound (US) and other imaging technique provides information about human anatomy. These technologies are more useful in diagnosis and treatment planning in medicine. Various methods can be applied to images obtained from these technologies to obtain essential features of images that help in diagnosis and treatment planning. Image segmentation is one such popular method in many image processing tasks. Segmentation is the process that segregates the pixels to bring out objects of interest in an image. A great variety of segmentations methods has been proposed in past decade $[2,3,30]$. Image segmentation plays an important role in biomedical-imaging applications. Medical applications of the segmentation is the study of human anatomy, to detect the region of interest, tumor burden etc. There are different problems in the segmentation of medical images such as intensity inhomogeneity, partial volume effect, artifacts, and closeness in grey level of different tissue [27]. Generally, medical images are complex in nature (diverse image content, cluttered objects, occlusion on-uniform object texture) and noisy, hence, making it difficult for segmentation. Further, boundary insufficiencies (i.e. missing edges and/or lack of texture contrast between regions of interest (ROIs) and background) make the segmentation task challenging. In this paper we present a brief overview of current segmentation methods [31] specifically used in detecting abnormalities in liver and abdomen CT scan images.

Several authors [6] have brought out survey of various segmentation techniques. However, comparative study of these methods has not been uniformly presented using a single database of medical images [7] thereby making it difficult for evaluation and comparison. The aim of this paper is to review segmentation techniques appeared in the recent literature on medical image segmentation and compare their performance by experimenting on liver and abdomen CT scan images of local database collected from PRISM Diagnostic Center, Solapur. Section II describes various image segmentation techniques in brief. Section III focuses on the experimental results of various segmentation algorithms. Finally, section IV presents conclusion.

\section{IMAGE SEGMENTATION AND CURRENT SEGMENTATION TECHNIQUES}

Image segmentation is the process of partitioning a digital image into multiple segments [2]. When applied to medical images, segmentation techniques identify the boundaries of objects such as organs or abnormal regions (e.g. tumors) in images. The resulting images enable for shape analysis, detecting volume change, and making a precise radiation therapy treatment plan. Various theoretical frameworks have been proposed for segmentation. Among some of the popular methods are Thresholding (Histogram thresholding and slicing techniques) [11,12], region growing (starting in the middle of an object and then "growing" outward until it meets the object boundaries) [10,14], edge detection and grouping (detected edges in an image are assumed to represent object boundaries) [6,7], active contour models and level sets (PDE frame work) [16,17], graph cut [ 32 ], and clustering [22, 23, 25, 26] (group together patterns that are similar in some sense.) Significant extensions and integrations of these frameworks [27] improve their efficiency, applicability and accuracy. The choice of segmentation techniques totally depends on the basis of type of image and type of problem domain being considered so that the results are acceptable by medical experts.

Different segmentation techniques are available in literature but not a single technique is useful for different types of images [4]. It is very difficult to develop a unified approach for image segmentation of different types of images and also it is very difficult to choose specific technique for a specific type of image. Therefore, image segmentation is a challenging problem in image processing. Due to different techniques, image segmentation is broadly classified in to two categories, on the basis of two properties of intensity values.

Detecting Discontinuities: This method is based on the intensity variation among the pixels [1]. Any significant changes in the intensity levels among neighboring pixels are termed as edges and results in the discontinuity in the pixels. It includes edge detection algorithms.

Detecting Similarities:-To partition an image in to regions on the basis of predefined criteria [1]. This method works on the 
basis of homogeneity criteria i.e. all pixels inside a region possess similar characteristics and dissimilar to the pixels in other region. It includes thresholding, region growing, region splitting and merging algorithms.

\subsection{Thresholding}

Thresholding is a simplest approach of segmentation having light object on dark background [1]. This method is based on the pixel values and image space region i.e. characteristics of images [7]. Threshold based technique divides the image into two classes; pixel belonging to certain range of intensity values represents one class and the rest of the pixels in the image represents the other class. It converts a multilevel intensity image into a binary image i.e. it choose a threshold $\mathrm{T}$, to divide image pixel into several regions and separate objects from background. If any pixel having intensity value is greater than or equal to threshold value then these pixels are considered as a part of object. i.e. $\mathrm{f}(\mathrm{x}, \mathrm{y}) \geq \mathrm{T}$, else pixel belong to background $[10,11]$. Mathematically, thresholding is represented as below

$$
\begin{aligned}
f(i, j) & =0 & , v(i, j)<L \\
& =1 & , v(i, j) \geq L
\end{aligned}
$$

Where, $v(i, j)$ is the pixel value at the position $(i, j)$.

Two variations of thresholding are common, global thresholding and local thresholding [12]. When the value of T is constant for the entire span of the image, thresholding is termed global, otherwise it is local thresholding. Global thresholding fails when background illumination is uneven. Multiple thresholds are used to avoid uneven illumination in local thresholding [9]. Thresholding does not yield results for multichannel images, and is sensitive to noise and intensity inhomogenities [7].

\subsection{Segmentation based on Edge Detection}

Edge detection methods are based on intensity variations among the pixels. Intensity data of an image provides uncertainly information about the location of edges [5]. This technique will resolve image segmentation by detecting edges between different regions that have discontinuity in intensity is extracted $[1,6]$. The boundaries of the two or more objects forms edges and major changes in the intensity levels between neighboring pixels in a certain direction are termed as edges and results in the discontinuity in the pixels. The resultant image is a binary image [7, 8]. Uncertainty of edges occurs because of noise introduced in the imaging process and later in the transmission and sampling process. The simplest way to detect edges is to look for abrupt intensity change using first derivative and second derivative of the intensity. There are two main edge based segmentation methods [7].

\subsubsection{Gray Histogram Techniques}

The final result of edge detection method mainly depends on the value of threshold T. It is very difficult to detect maximum and minimum gray histogram due to uneven impact of noise. Therefore, this method substitutes the curves of object and background with two conic Gaussian curves [7].

\subsubsection{Gradient based method}

The edge based techniques are mainly used to detect the object boundaries by using an edge detection operator and edge information [28]. When there is abrupt change in intensity near edge and little image noise then this method works better and it uses convolving gradient operator [7]. First edges are identified and linked close boundaries of the region to form required boundaries. Some common edge detection operators are used in this method such as Sobel operator, Laplace operator, Canny operator, Laplace of Gaussian operator and so on. Canny edge operator is most promising among all edge based operators but it takes more time as compared to Sobel operator [1].The main drawback of edge detection techniques is the presence of noise that results in random variation in the level from pixel to pixel [28].

Edge Detection algorithm are best suited for simple and noise free images as well often produce missing edges or extra edges on complex and noisy images [9]

\subsection{Region based segmentation methods}

Based on the principle of homogeneity, neighboring pixel having similar characteristic are grouped into one region and dissimilar characteristics are in to other region. A region of an image is defined as a connected homogeneous subset of the image with respect to some criteria $[1,13]$. This method is simple and more immune to noise [4, 7]. Region based methods are described below.

\subsubsection{Region growing method}

Region growing is one of the most popular techniques for segmentation due to simplicity and good performance. The method groups pixels into larger regions based on predefined criteria $[10,14]$. It starts with a set of initial seed points and grows the region. Seeds can be selected manually or automatically. Automatic selection of seed points is based on finding pixels that are of interest. Region growing algorithm is presented below.

1. Select seed pixels within the image [4].

2. Select similarity criteria on the basis of grey level intensity or color.

3. Appending each seed with neighboring pixels having predefined properties similar to seed pixel in to the region.

4. Repeat step iii) until no more pixels meet the criteria for allocation into the regions.

The main drawback of region growing is that it requires manual interaction to obtain the seed point. Region growing can also be sensitive to noise, causing extracted regions to have holes.

\subsubsection{Region splitting and merging}

In contrast to region growing, this method works on the entire image and segments the image on the basis of homogeneity criteria [15]. The method utilizes quad tree data to distinguish the homogeneity. It divides an image into the arbitrary unconnected regions and then merges the regions on the basis of condition of reasonable image segmentation $[2,7]$.

Initially, entire image is taken as a region and then the technique divides the entire region into four quadrants on the basis of predefined criteria. Repeating the process, it checks each of the quadrants, and divides into four quadrants for the same criteria. This procedure continues till the criteria satisfied or no further division is possible. Figure 1 illustrates the procedure. 

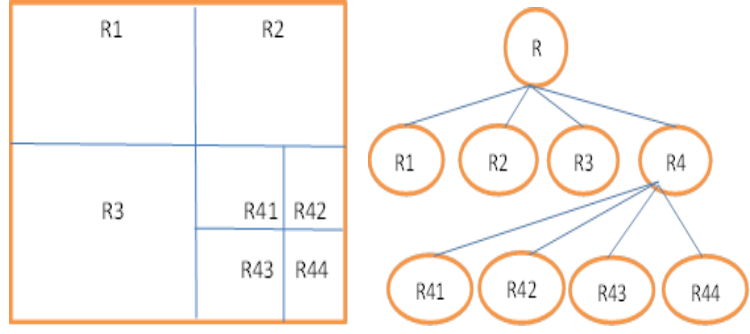

Fig. 1. Quad tree

The algorithm is presented below.

Let $\mathrm{R}$ represents the entire image region and $\mathrm{P}$ a predicate

1. If $\mathrm{P}(\mathrm{R})=\mathrm{FALSE} \quad[1]$, divides the image into quadrants. If $\mathrm{P}$ is FALSE for any quadrants i.e. $P\left(R_{i}\right)=$ FALSE then subdivide those quadrants into sub quadrants and so on till no further splitting is possible.

2. Merge any adjacent regions $R_{j}$ and $R_{k}$ for which, $\mathrm{P}\left(\mathrm{R}_{\mathrm{j}} \cup \mathrm{R}_{\mathrm{k}}\right)=$ TRUE.

3. Stop when no further merging is possible.

\subsection{Segmentation methods based on PDE (Partial Differential Equation)}

PDE image segmentation, introduced by Kassetal in 1987 [16], is mainly carried out using active contour or snakes methods. The method determines objects in presence of noise and ambiguities. Snake method transforms a segmentation problem into PDE framework and forwards to different methods of image segmentation such as, snake, level set and MumfordShah model.

\subsubsection{Snake Method}

Active contours and snake segmentation methods are computer generated curves which are used to find objects boundaries under the internal and external forces $[16,17]$. The work-in procedure is described below.

1. Snake is placed near the contour of Region of Interest (ROI)

2. Internal and External forces within the image control the shape and location of the snake within image [18].

3. The internal and external forces are used to calculate the contour of ROI by constructing energy function. The internal forces are used for smoothness while external forces guide the contours towards the contour of ROI.

The main drawback of snake method is that, it requires user interaction for curve drawing around detected objects [18]. Apart from this drawback, snake algorithm is sensitive to noise and computational complexity is high. Attempts have been made to solve these problems [ 16].

\subsubsection{Levelset Model}

This method developed by Osher and Sethian [16] is very useful on moving curves and surfaces with curvature based velocities. It represents the curves as the zero levelset of higher dimensional hyper surface. The method provides accurate numerical implementation and manages topological change. Levelset procedure is described below.
1) Pick up a set of seed points that represent an initial contour. It works for one seed point picked up manually or automatically. But, the less the seed points mask, the more the expensive calculations.

2) Create a signed distance function.

3) Compute feature image using gradient and gaussian filter.

4) Get the curve's narrow band.

5) Get curvature and use gradient descent to minimize energy.

6) Evolve the curve

7) Repeat the second step until obtaining the segmented region.

The main advantage of levelset is its stability; solve the problem of corner point producing, curve breaking and combining etc. The main drawback of this method is that, objects with edges defined by gradient can be segmented and curve may pass through object boundaries.

\subsection{Segmentation based on Artificial Neural Network (ANN)}

ANNs are widely used in medical image segmentation in recent years. Artificial representation of human brain that tries to simulate its learning process is called as neural net $[19,20$, $21]$. The neural network is trained with the sample set and this knowledge is used to segment the input image.

In this method, images are mapped into neural network. Image segmentation problem is converted into energy minimization problem where every neuron stands for a pixel $[6,7]$. Neural network segmentation consists of two steps based on neural network- feature extraction and image segmentation. Some features are extracted from the images, such that they are suitable for segmentation and these are the inputs of the neural network [17]. The basic structure of a neuron is described as shown in Fig. 2 where $X\left\{X_{i}, i=1,2, \cdots n\right\}$ represents the input to the neurons and $\mathrm{Y}$ represents the output. Each input is multiplied by its weight $W_{i}$, a bias $b$ is associated with each neuron. Each neuron has one pixel in an input image, receiving its corresponding pixels color information as an external stimulus. Also each neuron connects with its neighboring neurons, receiving local stimuli from them. The external and local stimuli are combined in an internal activation system, which accumulates the stimuli until it exceeds a dynamic threshold, resulting in a pulse output. The temporary series of pulse output shows information of input images and utilized for many image processing application, such as image segmentation and feature generation.

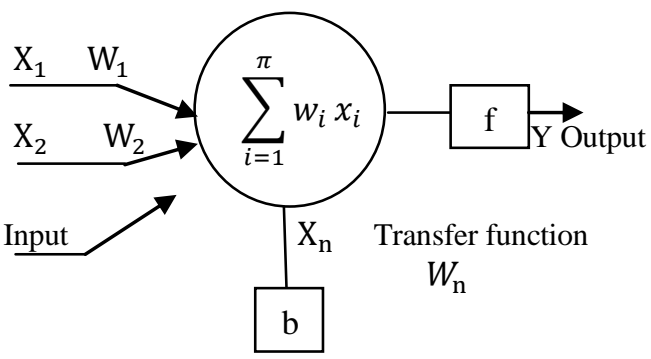

Fig. 2. A typical ANN structure.

This segmentation method relies on processing small areas of an image using artificial neural network or a set of neural networks. Many different neural network architectures are 
available such as Feedforward Network, Radial Basis Function Network, Feedback Network, and Self Organizing Map [29]. The method reduces manual expert intervention during image segmentation. The drawbacks of the method includes,

- Some predefined information is needed before segmentation.

- Initialization process makes influence to the result of image segmentation.

- Neural network uses trained sample set using learning process beforehand [7]. Therefore, period of training may be long so we should avoid overtraining.

\subsection{Segmentation based on clustering}

Clustering is an unsupervised learning task. It mainly used to identify a finite set of categories known as clusters to classify pixels [22]. It is mainly used when advance knowledge of classes is known. A predefined similarity criterion is defined between pixels $[2,3]$. Clusters are formed by grouping similar pixels. The formation of cluster is based on principle of maximizing the intra class similarity. Clustering is of different types such as, hard clustering, k-means clustering, fuzzy clustering etc.

\subsubsection{Hard Clustering}

Hard clustering consists of accurate boundaries between different clusters [23]. A pixel is belongs to only one cluster. A well-known example of hard clustering algorithm is K-means clustering algorithm [22, 23]. K-means clustering algorithm partition $\mathrm{n}$ pixels in to $\mathrm{k}$ clusters, where $\mathrm{k}<\mathrm{n}$. K-means algorithm is based on similarity and dissimilarity between pair of data component. K- mean algorithm classify pixels in an image into $\mathrm{k}$ number of clusters on the basis of similarity feature like grey level intensity of pixels and distance of pixel intensities from centroid pixel intensity[2]. Cluster can be selected manually, randomly or based on some conditions. Distance between the pixel and cluster center is measured by the squared or absolute difference between a pixel and a cluster center. This difference calculation is depends on pixel color, intensity, texture and location or weighted combination of these factors. The final output of the clustering method depends on the initial set of clusters.

The main advantage of K-means algorithm is simple and minimum execution cost. The main drawback of K-means algorithm is the determination of K, number of cluster [24] and it cannot give the same result every time when algorithm is executed. Resulting cluster heavily depend on the initial assignment of centroids.

\subsubsection{Fuzzy Clustering}

It is very difficult to classify the pixel in an image correctly [26] when there are no boundaries between objects in an image. To remove this problem fuzzy clustering method is used. Fuzzy clustering method classifies pixel values accurately, so it is broadly used in decision oriented applications such as tumor detection, tissue classification. Fuzzy clustering classifies the pixels into clusters based on similarity criteria i.e. distance; connectivity, intensity and pixels belong to some cluster. Fuzzy clustering algorithm includes FCM (Fuzzy C- Means), GK (Gustafson - kessel), GMD (Gaussian mixture decomposition), FCV (Fuzzy C varieties), AFC, FCS, FCSS, FCQS, FCRS etc.

\section{EXPERIMENTAL RESULTS}

The number of techniques for medical image segmentation is quite large and as a result focused on current techniques rather than being exhaustive. The techniques described in previous section are experimented on abdomen and liver CT images. The results are presented below.

\subsection{Thresholding}

A threshold is used turn a grey-scale image into a binary image. Threshold segmentation for the normal abdomen and liver CT is shown in Fig. 3. A threshold value of 0.39 is chosen on the basis of trial and error method. Threshold segmentation of the CT abdomen and liver image consisting of abnormality is shown in Fig. 4.

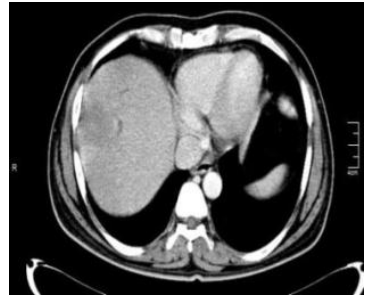

(a) Original image Fig. 3. Threshold segmentation of normal CT image.

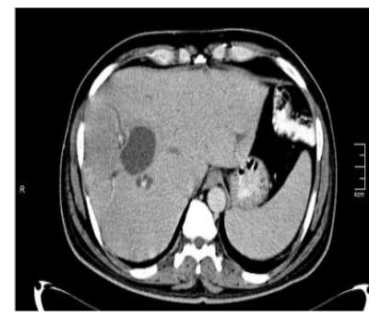

(a)
Original image

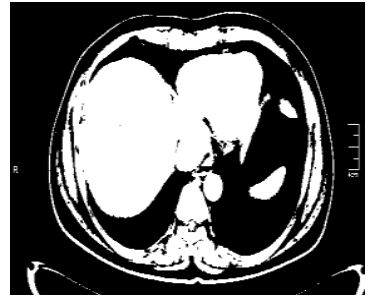

(b) Segmented image

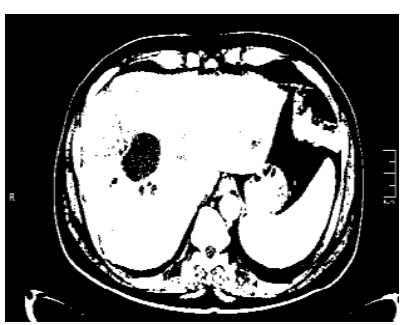

(b) Segmented image
Fig. 4. Threshold segmentation of abnormal CT image.

Adaptive threshold segmentation of the normal and abnormal abdomen and liver CT is shown in Fig. 5 and Fig. 6 respectively.

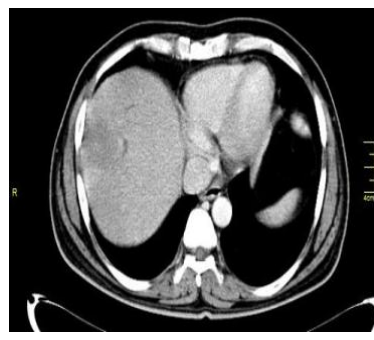

(a)
Original image

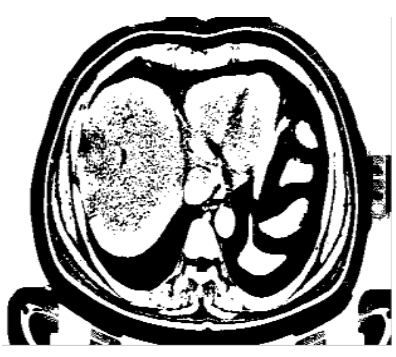

(b) Segmented image
Fig. 5. Adaptive Threshold segmentation of normal CT image. 


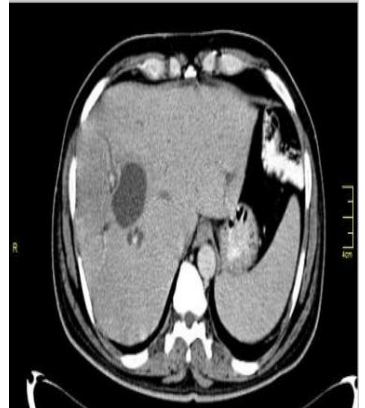

(a)

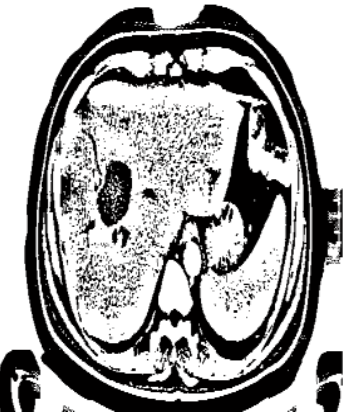

(b) Segmented image
Fig. 6. Adaptive Threshold segmentation of abnormal CT image.

Otsu's method: - Otsu's method selects the threshold by minimizing the within class variance of the two groups of pixels separated by the thresholding operator which in turn maximizes the between class variance. The result of applying Otsu's method for normal and abnormal abdomen and liver CT are shown in Fig. 7 and Fig. 8.

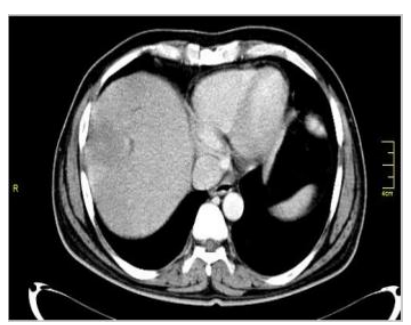

(a) Original image

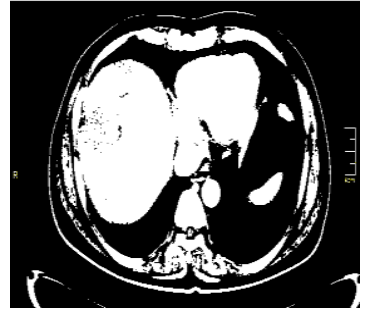

(b) Segmented image
Fig. 7. Otsu's Threshold segmentation of normal CT image.

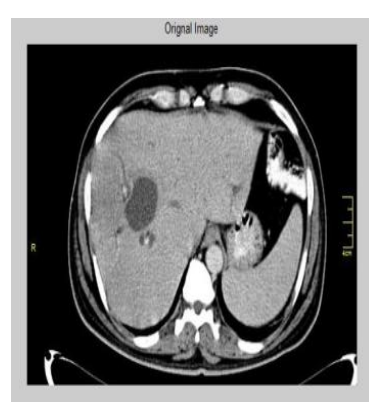

(a) Original image

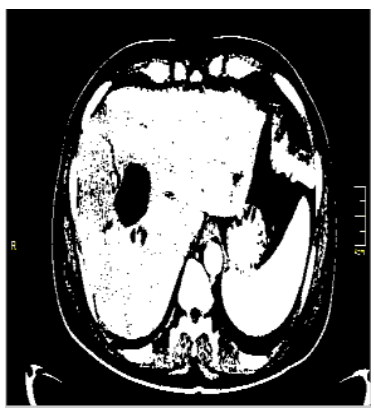

(b) Segmented image
Fig. 8. Otsu's Threshold segmentation of abnormal CT image.

\subsection{Region Growing}

This is a classical segmentation method. The seed mark each of the objects to be segmented. Five seed point are used in segmentation of the normal abdomen and liver CT images. The result is shown in Fig. 9 and Fig. 10 for both normal and abnormal CT images, respectively.

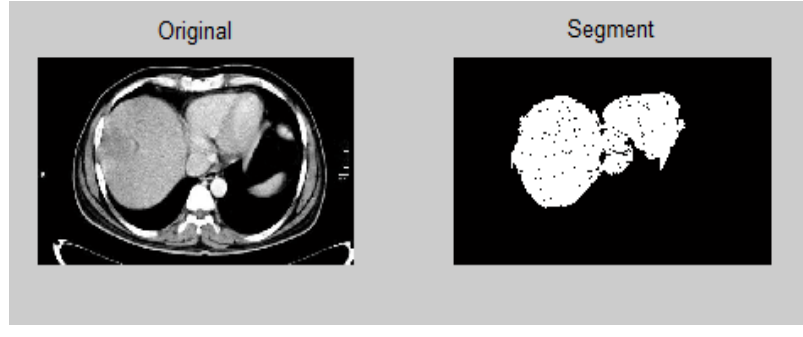

$\begin{array}{ll}\text { (a) Original image } & \text { (b) Segmented image }\end{array}$

Fig. 9. Region growing segmentation of normal CT image.

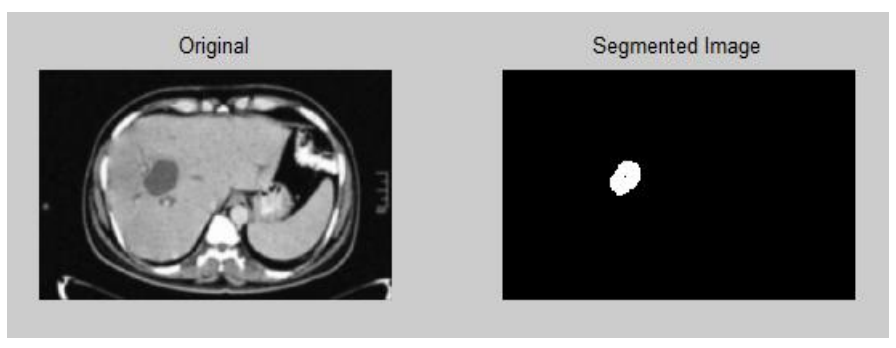

$\begin{array}{ll}\text { (a) Original image } & \text { (b) Segmented image }\end{array}$

Fig. 10. Region growing segmentation of abnormal CT image.

Region growing method gives better result as compared to the thresholding method. Region growing clearly extract the abnormality tissue area. However, the method requires manual interaction for the selection of seed point.

\subsection{Clustering}

The result of segmentation based on clustering is shown in Fig. 11 and Fig. 12 respectively.

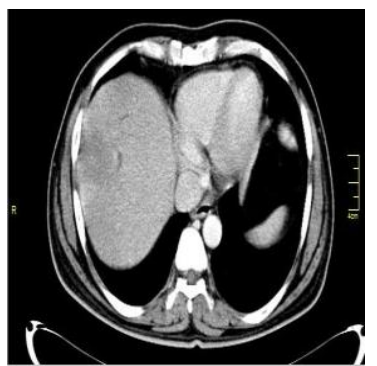

(a)
Original image

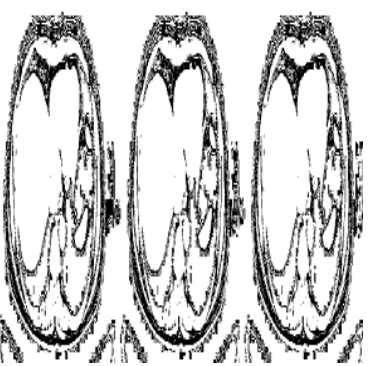

(b) Segmented image
Fig. 11. Clustering segmentation of normal CT image.

In clustering, there are many pixels missing as compared to the threshold method and the region growing method. Missing of pixels is due to noise interference and this lead to the holes in the segmented image. The result in Fig. 12 clearly indicates the abnormality part in abdomen and liver CT and satisfies the clinical validation. 


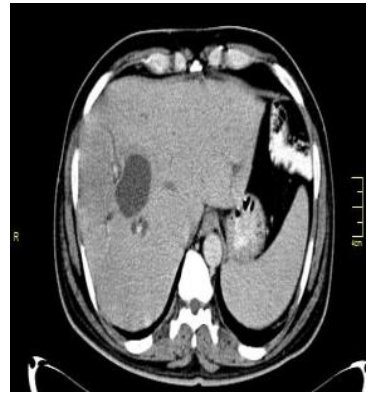

(a) Original image

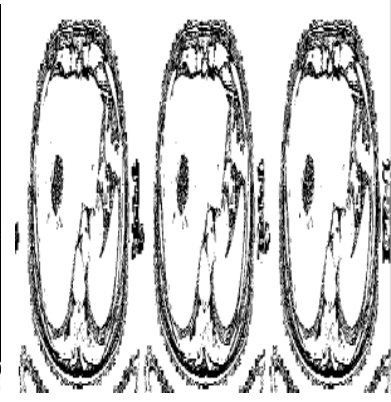

(b) Segmented image
Fig. 12. Clustering segmentation of abnormal CT image.

\subsection{Edge detection}

Edge detection method detects the object boundaries by using edge detection operator and extract boundaries by using the edge information. Canny edge and Sobel operators are used for edge detection. The results are shown in Fig. 13 and Fig. 14, respectively for normal and abnormal CT images. The complexity of medical images makes the correct boundary detection very difficult.

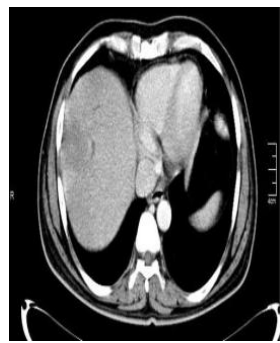

(a)Original image

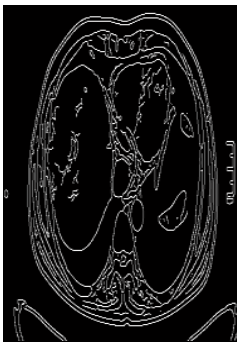

(b) Canny edge

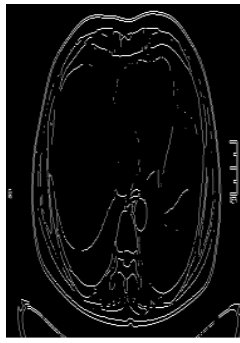

(c) Sobel edge
Fig. 13. Edge Detection based segmentation of normal CT image.

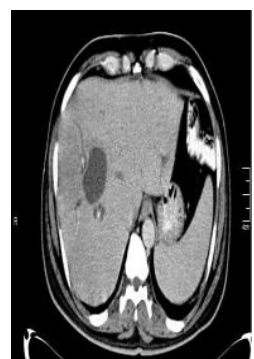

(a) Original image

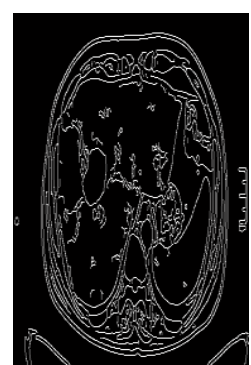

(b) Canny edge

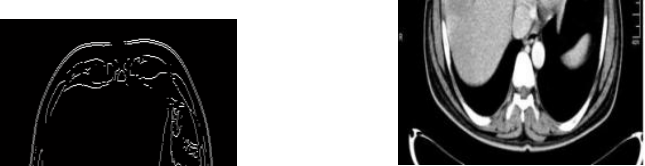

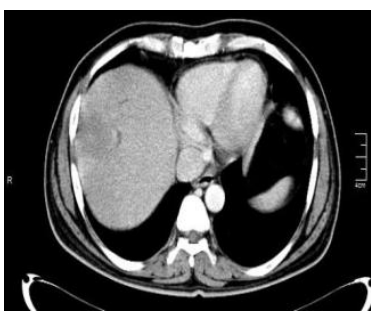

(a) Original image

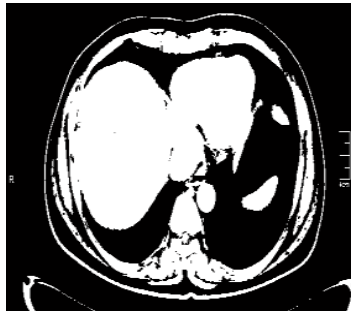

(b) Segmented image
Fig. 15. Levelset based segmentation of normal CT image.

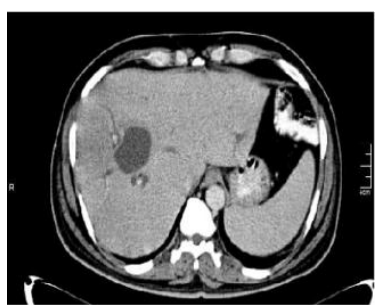

(a) Original image

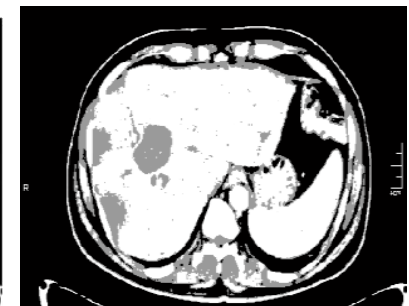

(b)

Segmented mage

Fig. 16. Levelset based segmentation of abnormal CT image.

\subsection{Region split and merging}

Split and merge is a region based segmentation technique that defines a predicate $\mathrm{R}$ which is the basis of splitting and merging. The result of applying region split and merging method for normal and abnormal abdomen and liver CT are shown in Fig. 17 and Fig. 18, respectively. (a)

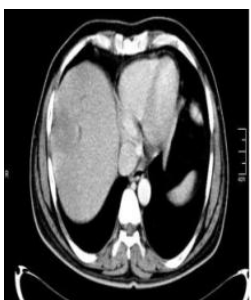

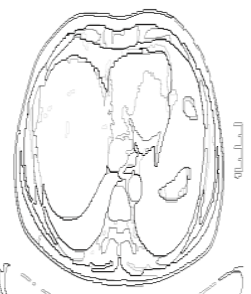

(b)

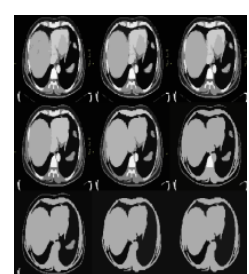

(c)
Fig. 17. Region Split and Merge segmentation of normal CT (a) Original image (b) normal segmented image (c) segmented image.

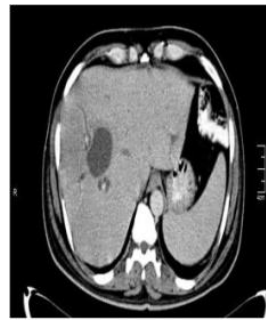

(a)

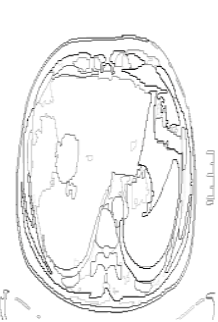

(b)

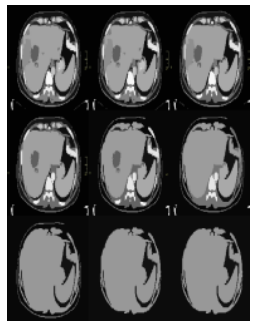

(c) that is evolved to the image contour. Result of applying Levelset method for normal and abnormal abdomen and liver CT are shown in Fig. 15 and Fig. 16, respectively.
Fig. 18. Region Split and Merge segmentation of abnormal CT (a) Original image (b) segmented image (c) abnormal segmented image. 


\subsection{Chan-vese active contour}

It performs active contour without edges on gray scale, binary image with initial phase mask. In order to define a user mask, the user should make sure that the dimensions of mask match those of the input image. The result of applying Chan-vese active contour method for normal and abnormal abdomen and liver CT are shown in Fig. 19 and Fig. 20 respectively.
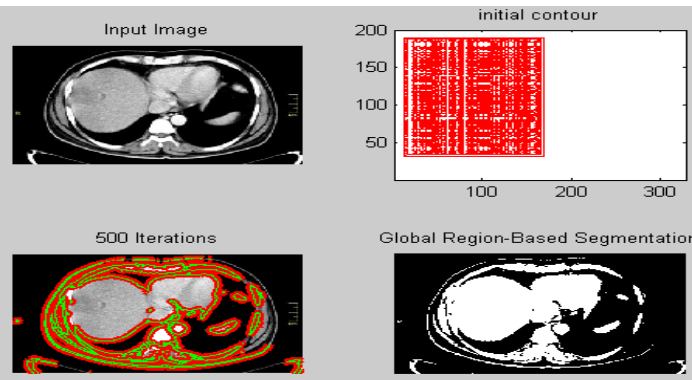

Fig. 19. Chen-vese segmentation of normal CT image.

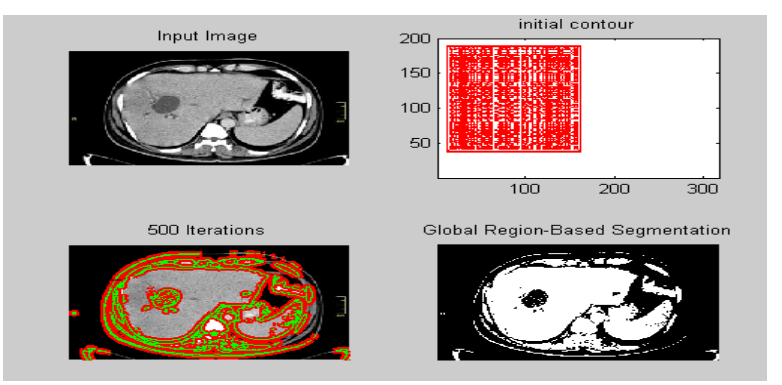

Fig. 20. Chen-vese segmentation of abnormal CT image.

Mean, standard deviation, and pixel values are computed for both normal and abnormal CT scan images. The values, correct to 4 decimal places, are presented in TABLE I and TABLE II for the segmentation methods studied.

TABLE I. parameter values for the segmented normal abdomen and liver CT.

\begin{tabular}{|l|l|l|l|l|}
\hline $\begin{array}{c}\text { Sr. } \\
\text { No. }\end{array}$ & \multicolumn{1}{|c|}{ Method } & \multicolumn{1}{|c|}{ Mean } & $\begin{array}{c}\text { Standard } \\
\text { Deviation }\end{array}$ & $\begin{array}{c}\text { Pixel } \\
\text { Values }\end{array}$ \\
\hline 1 & Thresholding & 71.4425 & 79.1362 & 52836 \\
\hline 2 & $\begin{array}{l}\text { Adaptive } \\
\text { thresholding }\end{array}$ & 67.2618 & 46.6472 & 106072 \\
\hline 3 & Region growing & 87.2836 & 32.8806 & 137647 \\
\hline 4 & Clustering & 81.2850 & 38.3532 & 383795 \\
\hline 5 & Canny edge & 8.7432 & 28.1380 & 13891 \\
\hline 6 & Sobel edge & 3.1688 & 17.4709 & 5005 \\
\hline 7 & Levelset & 43.1340 & 35.9951 & 67865 \\
\hline 8 & $\begin{array}{l}\text { Region split and } \\
\text { merge }\end{array}$ & 32.7954 & 144.1601 & 51816 \\
\hline 9 & Otsu's method & 76.3905 & 114.7954 & 421753 \\
\hline 10 & $\begin{array}{l}\text { Chen-Vese active } \\
\text { contour }\end{array}$ & 32.5973 & 46.5281 & 20912 \\
\hline
\end{tabular}

TABLE II. Parameter values for the segmented abnormal abdomen and liver CT (ROI).

\begin{tabular}{|l|l|l|l|l|}
\hline $\begin{array}{c}\text { Sr. } \\
\text { No. }\end{array}$ & \multicolumn{1}{|c|}{ Method } & \multicolumn{1}{|c|}{ Mean } & $\begin{array}{c}\text { Standard } \\
\text { Deviation }\end{array}$ & \multicolumn{1}{|c|}{$\begin{array}{c}\text { Pixel } \\
\text { Values }\end{array}$} \\
\hline 1 & Thresholding & 74.1348 & 79.1988 & 55832 \\
\hline 2 & $\begin{array}{l}\text { Adaptive } \\
\text { thresholding }\end{array}$ & 68.8537 & 46.0686 & 109805 \\
\hline 3 & Region growing & 86.5108 & 33.7665 & 138200 \\
\hline 4 & Clustering & 81.6685 & 37.8246 & 390349 \\
\hline 5 & Canny edge & 8.7915 & 28.2460 & 14183 \\
\hline 6 & Sobel edge & 3.1071 & 17.3142 & 5000 \\
\hline 7 & Levelset & 45.7904 & 36.8220 & 73224 \\
\hline 8 & $\begin{array}{l}\text { Region split and } \\
\text { merge }\end{array}$ & 31.8325 & 141.5739 & 51352 \\
\hline 9 & Otsu's method & 86.2060 & 118.5807 & 486085 \\
\hline 10 & $\begin{array}{l}\text { Chen-Vese active } \\
\text { contour }\end{array}$ & 35.1068 & 47.3675 & 22003 \\
\hline
\end{tabular}

Further, mean computed using machine (Siemens somatom scope 16 slice) were noted down for the same set of images used for experimenting. The machine generated mean were compared with mean obtained with our experimental results and are tabulated in TABLE III and TABLE IV for normal and affected images respectively. From the tables, it is evident that Region growing method gave better results followed by Otsu's method compared to other segmentation methods.

TABLE III. Mean values measured for the normal abdomen and liver CT by Machine.

\begin{tabular}{|c|c|c|c|c|}
\hline $\begin{array}{c}\text { Sr. } \\
\text { No. }\end{array}$ & Method & $\begin{array}{c}\text { Computed } \\
\text { Mean }\end{array}$ & $\begin{array}{c}\text { Mean } \\
\text { measured } \\
\text { by } \\
\text { machine }\end{array}$ & $\begin{array}{c}\text { Difference } \\
\text { Mean }\end{array}$ \\
\hline 1 & Thresholding & 71.4425 & 110 & 38.557 \\
\hline 2 & $\begin{array}{c}\text { Adaptive } \\
\text { thresholding }\end{array}$ & 67.2618 & 110 & 42.738 \\
\hline 3 & $\begin{array}{c}\text { Region } \\
\text { growing }\end{array}$ & 87.2836 & 110 & 22.716 \\
\hline 4 & $\begin{array}{c}\text { Clustering } \\
\text { Canny edge }\end{array}$ & 81.2850 & 110 & 28.715 \\
\hline 5 & $\begin{array}{c}\text { Sobel edge } \\
\text { Levelset }\end{array}$ & 43.1688 & 110 & 106.831 \\
\hline 7 & $\begin{array}{c}\text { Region split } \\
\text { and merge }\end{array}$ & 32.7954 & 110 & 77.204 \\
\hline 9 & Otsu's method & 76.3905 & 110 & 30.609 \\
\hline 10 & $\begin{array}{c}\text { Chen-Vese } \\
\text { active contour }\end{array}$ & 32.5973 & 110 & 77.402 \\
\hline
\end{tabular}


TABLE IV. Mean values measured for the abnormal abdomen and liver CT by Machine.

\begin{tabular}{|c|l|c|c|c|}
\hline $\begin{array}{c}\text { Sr. } \\
\text { No. }\end{array}$ & \multicolumn{1}{|c|}{ Method } & $\begin{array}{c}\text { Computed } \\
\text { Mean }\end{array}$ & $\begin{array}{c}\text { Mean } \\
\text { measured } \\
\text { by } \\
\text { machine }\end{array}$ & $\begin{array}{c}\text { Difference } \\
\text { Mean }\end{array}$ \\
\hline 1 & Thresholding & 74.1348 & 114 & 39.865 \\
\hline 2 & $\begin{array}{l}\text { Adaptive } \\
\text { thresholding }\end{array}$ & 68.8537 & 114 & 45.146 \\
\hline 3 & Region growing & 86.5108 & 114 & 27.489 \\
\hline 4 & Clustering & 81.6685 & 114 & 32.332 \\
\hline 5 & Canny edge & 8.7915 & 114 & 105.209 \\
\hline 6 & Sobel edge & 3.1071 & 114 & 110.893 \\
\hline 7 & Levelset & 45.7904 & 114 & 68.210 \\
\hline 8 & $\begin{array}{l}\text { Region split and } \\
\text { merge }\end{array}$ & 31.8325 & 114 & 82.168 \\
\hline 9 & Otsu's method & 86.2060 & 114 & 27.794 \\
\hline 10 & $\begin{array}{l}\text { Chen-Vese active } \\
\text { contour }\end{array}$ & 35.1068 & 114 & 78.893 \\
\hline
\end{tabular}

\section{CONCLUSION}

Image segmentation plays an important role in medical imaging, by automating or providing the facility of the delineation of anatomical structures and region of interest. In this paper, several current segmentation techniques used in medical imaging has been studied and experiments are performed on liver and CT images for performance comparison. Outcomes of the segmentation techniques which are discussed in this paper depends on some input parameter like threshold for the thresholding, number of cluster centers, seed point for the region growing. Running time for the clustering method depends on the number of iteration used and edge detection depends on discontinuity of grey level or intensity variation on the grey scale images. Region growing needs manual interaction. If the threshold value is accurate then thresholding gives best performance. From experimental results it is evident that region growing method performed better with results comparable with machine generated values (mean value) than the other segmentation techniques. Our future study is to combine existing techniques with preprocessing procedures and volume estimation in order to improve the results in segmentation.

\section{REFERENCES}

[1] Rafael C. Gonzalez, Richard E. Woods, "Digital Image Processing", 2nd ed., Beijing: Publishing House of Electronics Industry, 2007.

[2] S.Aksoy, "Image Segmentation", Department of Computer Engineering, Bilkent Univ.

[3] Zhang, Y. J, An Overview of Image and Video Segmentation in the last 40 years, Proceedings of the 6th International Symposium on Signal Processing and Its Applications, pp. 144-151, 2001.

[4] K. K. Singh, A. Singh,"A Study of Image Segmentation Algorithms for Different Types of Images", International Journal of Computer Science Issues, Vol. 7, Issue 5, 2010.
[5] Jesmin F. Khan, Sharif M. A. Bhuiyan, and Reza R. Adhami," Image Segmentation and Shape Analysis for Road-Sign Detection", IEEE TRANSACTIONS ON INTELLIGENT TRANSPORTATION SYSTEMS, VOL. 12, NO. 1, MARCH 2011.

[6] N. R. Pal, S. K. Pal,“A Review on Image Segmentation Techniques”, Pattern Recognition, Vol. 26, No. 9, pp. 1277- 1294, 1993.

[7] W. X. Kang, Q. Q. Yang, R. R. Liang,"The Comparative Research on Image Segmentation Algorithms", IEEE Conference on ETCS, pp. 703-707, 2009.

[8] Rastgarpour M., and Shanbehzadeh J., Application of AI Techniques in Medical Image Segmentation and Novel Categorization of Available Methods and Tools, Proceedings of the International MultiConference of Engineers and Computer Scientists 2011 Vol I, IMECS 2011, March 16-18, 2011, Hong Kong.

[9] S. S. Varshney, N. Rajpal, R. Purwar,"Comparative Study of Image Segmentation Techniques and Object Matching using Segmentation", Proceeding of International Conference on Methods and Models in Computer Science, pp. 1-6, 2009.

[10] K. G. Gunturk, "EE 7730- Image Analysis I", Louisiana state university.

[11] L.Aurdal,"Image Segmentation beyond thresholding”, Norsk Regnescentral, 2006.

[12] Y. Zhang, H. Qu, Y. Wang,“Adaptive Image Segmentation Based on Fast Thresholding and Image Merging", Artificial reality and Telexistence-Workshops, pp. 308-311, 1994.

[13] H. G. Kaganami, Z. Beij, “Region Based Detection versus Edge Detection", IEEE Transactions on Intelligent information hiding and multimedia signal processing, pp. 1217-1221, 2009.

[14] Y. Chang, X. Li, “Adaptive Image Region Growing”, IEEE Trans. On Image Processing, Vol. 3, No. 6, 1994.

[15] Bo Peng, Lei Zhang, and David Zhang, "A Survey of Graph Theoretical Approaches to Image Segmentation".

[16] X. Jiang, R. Zhang, S. Nie,"Image Segmentation Based on PDEs Model: a Survey", IEEE conference, pp. 1-4, 2009.

[17] C. Zhu, J. Ni, Y. Li, G. Gu, "General Tendencies in Segmentation of Medical Ultrasound Images", International Conference on ICICSE, pp. 113-117, 2009.

[18] P. Karch, I. Zolotova, “An Experimental Comparison of Modern Methods of Segmentation", IEEE 8th International Symposium on SAMI, pp. 247-252, 2010.

[19] T.F. Wang, D.Y. Li et al. Automatic segmentation of medical ultrasound image using self-creating and organizing neural network.Journal of electronics.1999,21(1),pp.124-127.

[20] Z. B. Chen, Q. H. Zheng, T. S. Qiu, Y. Liu. A new method for medical ultrasonic image segmentation.Chinese Journal of Biomedical Engineering.2006, 25(6), pp.650-655.

[21] Y.L.Huang,D.R.Chen. Watershed segmentation for breast tumor in 2Dsonography. Ultrasound in Medicine \& Biology. 2004, 30(5), pp.625632. 
[22] V. K. Dehariya, S. K. Shrivastava, R. C. Jain,“Clustering of Image Data Set Using K-Means and Fuzzy K-Means Algorithms", International conference on CICN, pp. 386391, 2010.

[23] F .Z. Kettaf, D. BI, J. P., “A Comparison Study of Image Segmentation by Clustering Technique", Proceedings of ICSP, pp. 1280-1282, 1996.

[24] P.Lukac, R. Hudec, M. Benco, P. Kamencay, Z. Dubcova, M. Zachariasova,"Simple Comparison of Image Segmentation Algorithms Based on Evaluation Criterion”, IEEE Conference on Radioelektronika, pp. 14, 2011.

[25] S.Tatiraju, A. Mehta, "Image Segmentation using kmeans clustering, EM and Normalized Cuts", Department of EECS, pp. 1-7.

[26] S. Naz, H. Majeed, H. Irshad,"Image Segmentation using Fuzzy Clustering: A Survey", International Conference on ICET, pp.181-186, 2010.

[27] Dzung L. Pham, ChenyangXu, and Jerry L. Princ,"Current Methods In Medical Image Segmentation," Department of Electrical and Computer Engineering, The Johns Hopkins University,Annu. Rev. Biomed. Eng. 2000. 02:315-37.
[28] Krit Somkantha, Nipon Theera-Umpon and Sansanee Auephanwiriyakul," Boundary Detectioon in Medical Images Using Edge Following Algorithm Based on Intencity Gradient and Texture Gradient Features" IEEE transaction on biomedical engineering, vol. 58, no. 3 , march 2011.

[29] J.Jiang, p. Trundle and J. Ren, Digital Media and Systems Research Institute, University of Bradford," Medical Image Analysis with Artificial Neural Networks."

[30] Rajeshwar Dass, Priyanka, Swapna devi," Image Segmentation Techniques", IJECT vol. 3, march 2012.

[31] A.M Khan, Ravi S," Image Segmentation Methods: A Comparative Study" International Journal of Soft Computing and Engineering (IJSCE), Vol-3, Issue-4, September 2013.

[32] Christo Ananth, Karthika, Shivangi singh, Jennifer Christa, Gracelyn Ida," Graph Cutting Tumor Images" International Journal of Advanced Research in Computer Science and Software Engineering, vol 4, Issue 3, march 2014. 